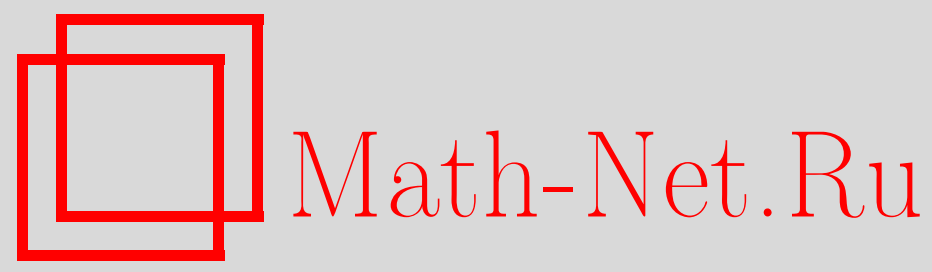

А. И. Созутов, Н. М. Сучков, О бесконечных группах с заданной сильно изолированной 2-подгруппой, Матем. заметки, 2000, том 68, выпуск 2, 272-285

DOI: https://doi.org/10.4213/mzm944

Использование Общероссийского математического портала Math-Net.Ru подразумевает, что вы прочитали и согласны с пользовательским соглашением http://www . mathnet.ru/rus/agreement

Параметры загрузки:

IP : 54.237 .59 .107

26 апреля 2023 г., $16: 24: 10$






\title{
О БЕСКОНЕЧНЫХ ГРУППАХ \\ С ЗАДАННОЙ СИЛЬНО ИЗОЛИРОВАННОЙ 2-ПОДГРУППОЙ
}

\author{
А. И. Созутов, Н. М. Сучков
}

Доказана локальная конечность некоторых групп с сильно изолированной 2-подгруппой периода, не превосходящего 4. В частности, дан положительный ответ на вопрос 10.76 Шункова из Коуровской тетради.

Библиографияя: 18 названий.

В настоящей заметке завершается решение вопроса 10.76 Шункова из Коуровской тетради [1, теорема 2 и следствие 1], анонсированное в [2], [3]. Сформулируем этот вопрос.

Вопрос. Пусть $G$ - периодическая группа, обладающая бесконечной силовской 2-подгруппой $S$, которая либо әлементарная абелева, либо 2-группа Сузуки, причем нормализатор $N_{G}(S)$ сильно вложен в $G$ и является группой Фробениуса $c$ локально ииклическим дополнением. Долэсна ли әруппа $G$ быть локально конечной?

Для случая, когда $S$ - элементарная абелева 2-подгруппа, положительное решение вопроса было получено в [4]. Теорема 1 нашей заметки дополняет результат из [4].

Определим 2-әруппу Судзуки $S(P, x)$ [5]. Пусть $P$ - поле характеристики 2, допускающее нетривиальньй автоморфизм $x$ такой, что если $\alpha^{1+x}=1$, то $\alpha=1$. Пусть $U=S(P, x)=\{(\alpha, \beta) \mid \alpha, \beta \in P\}$ и $(\alpha, \beta) \cdot(\gamma, \delta)=\left(\alpha+\gamma, \alpha \gamma^{x}+\beta+\delta\right)$. Относительно этой операции $U-2$-группа. Если $x^{2}=2$, то $U$ изоморфна силовской 2 -подгрупе простой групшы Судзуки $S z(P)$.

Шунков пояснил авторам, что именно групу $S=S(P, x)$ он имел в виду, формулируя свой вопрос. Дело в том, что Хигман [6] кроме груп $S(P, x)$ назвал группами Судзуки некоторые центральные произведения групा вида $S(P, x)$ и, в частности, силовские 2 -подгруппы унитарных групп $U_{3}(P)$. Но в $U_{3}(P)$ силовская 2-подгруппа $S$ не является сильно изолированной (см., например, $[7$, c. 166]). Поэтому для таких силовских 2 -подгруп $S$ вопрос 10.76 (при условии существования групшы $G$ ) решается безусловно отрицательно.

Напомним некоторые определения.

Работа выполнена при финансовой поддержке Российского фонда фундаментальных исследований, грант № 99-01-00542. 
Собственная подгруппа $B$ групшы $G$ называется сильно вложснннй, если $B$ содержит элемент порядка 2 ( инволюиию) и для любого элемента $g \in G \backslash B$ подгрупша $B \cap B^{g}$ не содержит инволюций [7]. Элемент $а$ группы $G$ назьвается конечным, если при любом $g \in G$ подгруппа $\left\langle a, a^{g}\right\rangle$ конечна [8]. Подгруппа $H$ группы $G$ называется сильно изолированной, если для любого неединичного элемента $h \in H$ выполняется свойство $C_{G}(h) \leqslant H[9]$.

ТЕОрема 1. Пусть бесконечная группа $G$ содержит сильно изолированную 2-подәруппу $U$ периода, не превосходящего 4, конечную инволючию и подгруппу порядка 6. Тогда либо $G=F \lambda U$ - локально конечная группа Фробениуса с периодическим абелевым ядром $F$, либо $G$ изоморфна группе $L_{2}(Q)$ над подходящим локально конечным полем $Q$ характеристики 2.

ТЕОРема 2. Пусть бесконечная группа $G$ с конечной инволюиией содержит сильно изолированную подгруппу $U$, изоморфную 2-группе Судзуки $S(P, x)$. Тогда либо подгруппа $U$ нормальна в $G$, либо $G$ изоморфна группе Судзуки $S z(Q)$ над подходящим локально конечным полем $Q$ характеристики 2.

СлЕдСТВИЕ 1. Пусть $G$ - периодическая группа, обладающая бесконечной силовской 2-подгруппой $S$, которая либо элементарная абелева, либо 2-группа Сузу$\kappa и S(P, x)$, причем нормализатор $N_{G}(S)$ сильно вложен в $G$ и является группой Фробениуса с локально ииклическим дополнением. Тогда $G$ локально конечна $u$ изоморфна одной из әрупп $L_{2}(Q), S z(Q)$ над подходящим локально конечным полем $Q$ характеристики 2.

Мы будем существенно использовать результаты работ [5], [4], [10], [11] и некоторые, ключевые из них, приведем с доказательствами. Вместе с тем, ограниченность объема статьи - причина краткости ее изложения.

\section{1. Леммы о сильно вложенной подгруппе.}

ЛЕмма 1.1 (см. [4, леммы $1.7,2.1]$ ). Пусть $G$ - әруппа с сильно вложсенной подгруппой $B$ и конечной инволюиией. Тогда

1) все инволюиии в $G$ сопряжены и все инволюции из $B$ сопряжены в $B ; \boldsymbol{\theta}$ частности, любая пара инволючий в $G$ порождает конечную подгруппу;

2) между множеством инволюиий из $B$ и множеством инволюиий любого правого смежсного класса $B g$, где $g \in G$, моэкно установить взаимно однозначное соответствие; при этом если $i$ - фиксированная инволюция из $B$, то каждой инволюиии $k \in B$ (в том числе и инволючии $i$ ) соответствует единственная инволюиия $j_{k} \in B g$ такая, что $g^{-1} \mathrm{~kg}=j_{k} i j_{k}$;

3) любой әлемент $g \in G$ обладает представлением $g=h j$, где $h \in B, a j-$ некоторая инволюиия;

4) для любой инволюиии $j \in G \backslash B$ в подгруппе $B$ существует множество $M_{j}$ строго вещественных относительно $j$ әлементов той же мощности, что и множество инволюиий из $B$.

ЛЕмма 1.2 (см. [4, лемма 2.2]). Пусть $G$ - әруппа с сильно вложсенной подгруппой $B$ и конечной инволючией, $i \in B u j \in G \backslash B$ - произвольные инволючии, 
$H_{j}=B \cap B^{j} \quad$ u $T_{j}$ - подгруппа в $B$, порожсденная всеми строго вешественными относительно $j$ әлементами из В. Тогда

1) имеет место влохсение $T_{j} \leqslant H_{j}, H_{j}$ - подгруппа без инволюиий, $\left\langle j, T_{j}\right\rangle=$ $T_{j} \lambda\langle j\rangle u\left\langle j, H_{j}\right\rangle=H_{j} \lambda\langle j\rangle$

2) в каждом смежсном классе $C_{B}(i) \cdot b$, әде $b \in B$, существует единственный инвертируемый инволюиией $j$ әлемент и его порядок конечен и нечетен;

3) выполнено равенство $B=T_{j} \cdot C_{B}(i)=H_{j} \cdot C_{B}(i)$ u $i^{T_{j}}$ - мнохество всех инволюиий группь $B$;

4) если $b$ - неединичный строго вещественный относительно инволючии $j \in$ $G \backslash B$ әлемент из $B$, то $C_{B}(b)$ не содержит инволючий.

Всякое соотношение вида

$$
v t v=u^{-1} v u, \quad|t|=|v|=2, \quad|u|=q=2^{k}, \quad t u=u t,
$$

в группе с сильно вложенной подгруппой будем называть структурным тождеством Судзуки.

Лемма 1.3. Пусть $G$ - группа с сильно вложсенной подгруппой $B$ и конечной инволюиией, $t \in B, v \in G \backslash B$ - произвольнье инволюиии $и$ для тройки $(v, t, u)$ выполняются соотношения (1). Тогда $(v t)^{u}=(v t)^{2}$, порядок әлемента vт конечен $u$ нечетен, $L=\langle v, t, u\rangle$ - конечная группа Фробениуса с ядром $\langle v t\rangle$ и неинвариантным множителем $\langle u\rangle, u \in B u t \in\langle u\rangle$.

ДокАЗАтЕльство. Домножим равенство $v t v=u^{-1} v u$ справа на элемент $t$ и, воспользовавшись соотношением $t u=u t$, получим $v t v t=(v t)^{2}=u^{-1} v u t=u^{-1} v t u=(v t)^{u}$. Так как $|u|=q=2^{k}$ и $(v t)^{2^{q}}=u^{-q}(v t) u^{q}=v t$, то порядок элемента $v t$ конечен и нечетен.

Поскольку инволюции $v$ и $t$ инвертируют $v t$, то подгруппа $A=\langle v t\rangle$ нормальна в $L$, причем ввиду леммы 1.1 подгруппа $C_{L}(x)$ не содержит инволюций при любом неединичном $x \in\langle v t\rangle$. Таким образом, в $L$ нет четверных подгрупп и из соотношения $u t=t u$ вытекает $t \in S=\langle u\rangle$. Понятно, что $u \in B$, и очевидно, что $L=A \lambda S$ - конечная группа $\Phi$ робениуса. Лемма доказана.

\section{2. Леммы о нормализаторе сильно изолированной 2-подгруппы.}

Лемма 2.1. Пусть $G$ - группа с сильно изолированной 2-подгруппой $U$ и ко-

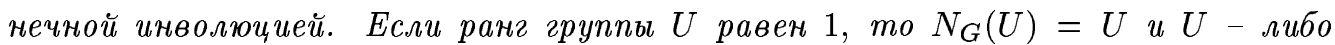
(локально) чиклическая группа, либо (обобщенная) группа кватернионов. Если, дополнительно, подгруппа $U$ конечна, то $G=F \lambda U$ - локально конечная группа Фробениуса с неинвариантным множителем $U$ и абелевым ядром $F$.

ДоКАЗАТЕЛЬСтво. По условиям леммы $U$ есть 2-групша с единственной инволюцией $i$. Значит, $N_{G}(U)=U=C_{G}(i)$ и $U$ - либо (локально) циклическая группа, либо (обобщенная) группа кватернионов [12]. Пусть $j$ - конечная инволюция из $G \backslash U$. Тогда $D=\langle i, j\rangle$ - конечная группа диэдра и ввиду сильной изолированности $U$ порядок элемента $i j$ нечетен. Следовательно, инволюции $i$ и $j$ сопряжены в $D$ и $i-$ конечная инволюция. Если $U=C_{G}(i)-$ конечная подгруппа, то $G$ локально конечна по основной 
теореме из [13]. Понятно, что $U \cap U^{g}=1$ для любого элемента $g \in G \backslash U$, и последнее утверждение леммы вытекает из известной теоремы Фробениуса для локально конечных групп [14]. Лемма доказана.

В следующих ниже леммах $G$ - группа с сильно изолированной 2-подгруппой $U$ и конечной инволюцией. Обозначим $B=N_{G}(U)$ и в дальнейшем будем предполагать, что $B \neq G$ и $r(U)>1$.

ЛЕмма 2.2. Пусть $v$ - произвольная инволюиия из $G \backslash B u H=B \cap B^{v}$. Справедливы следуюшие утверждения:

1) подгруппа $B$ сильно влохсена в $G$;

2) все инволюиии в $G$ сопряжены;

3) $B=U \lambda H$ и все инволюиии из $U$ содержатся в ее чентре $Z(U)$;

4) $H$ - периодическая локально ииклическая группа, инвертируемая инволюцией $v$ и действующая транзитивно на мнохсестве всех инволючий из $U$;

5) $\Omega_{1}(Z(U)) \lambda H$ изоморфна подгруппе Бореля группь $L_{2}(Q)$ над подходящим локально конечным полем $Q$ характеристики 2;

6) подгруппа $H$ сильно изолирована в $G$;

7) если $U-$ локально конечная группа, то $B=U \lambda H-$ группа Фробениуса.

ДокАЗАТЕЛЬСтво. 1) Легко убедиться, что все 2-элементы из $B$ содержатся в $U$. Пусть $g \in G$ и $T=U \cap U^{g} \neq 1$. Тогда подгруппа $T$ сильно изолирована в $G$. Отсюда выводим, что все инволюции из $U$ лежат в $T$, любая циклическая подгруппа из $U$ содержится в $T, T=U$ и $g \in B$. Таким образом, $2 \notin \pi\left(B \cap B^{g}\right)$ для любого элемента $g \in G \backslash B$. Утверждение доказано.

2) Утверждение вытекает из леммы 1.1 и утверждения 1) настоящей леммы.

3) По лемме 1.2 подгруппа $H$ не содержит инволюций и $B=H \cdot C_{B}(i)$, где $i$ - произвольная инволюция из $U$. Поскольку $C_{B}(i) \leqslant U$, то $B=U \lambda H$ и $C_{B}(i)=U$. Утверждение доказано.

4) Из сильной изолированности $U$ в $G$ и утверждения 2$)$ леммы следует, что $C_{H}(v)=1$ и $v$ - конечная инволюция в $H \lambda\langle v\rangle$. Поэтому для любого элемента $h \in H$ порядок элемента $v v^{h}$ конечен, нечетен и инволюции $v, v^{h}$ сопряжены в $\left\langle v, v^{h}\right\rangle$, что влечет $h \in\left\langle v v^{h}\right\rangle$ и $h^{v}=h^{-1}$. Таким образом, $H$ - периодическая группа без инволюций, инвертируемая инволюцией $v$, и $H$ - абелева группа. Как периодическая абелева группа регулярных автоморфизмов абелевой групшы $Z(U) H$ - локально циклическая группа [9], [14]. Утверждение доказано.

5) Утверждение доказано в [4, лемма 4.4].

6) Если $C_{G}(h) \neq H$ для некоторого элемента $h \in H^{\#}$, то по теореме 3.1 из [4] $r(U)=1$, противоречие. Следовательно, подгрупша $H$ сильно изолирована в $G$.

7) Как доказано выше, $B=U \lambda H$ и $H$ - периодическая локально циклическая групша. По теореме Шмидта [15] группа $B$ локально конечна, и понятно, что $H \cap H^{g}=1$ для любого элемента $g \in B \backslash H$. По теореме $\Phi$ робениуса для локально конечных групп [14] $B$ - группа Фробениуса с неинвариантным множителем $H$ и ядром $U$. Лемма доказана.

Обозначим через $\Omega$ множество всех подгрупп из $G$, сопряженных с подгруппой $B$. Далее будем рассматривать подстановочное представление группы $G$ на множестве $\Omega$, при этом обозначим $B=\alpha \in \Omega$. 
Лемма 2.3. Пусть $B=U \lambda H$ - группа Фробениуса с неинвариантным множителем $H$ и ядром $U, H=B \cap B^{v}$, где $v-$ инволюиия из $G \backslash B$. Тогда

1) $v^{B}$ - мнохсество всех инволюиий из $G \backslash B$;

2) группа $G$ 2-транзитивна на $\Omega$, при этом $B=G_{\alpha}-$ стабилизатор точки и $H=G_{\alpha \beta}-$ стабилизатор двух точек;

3) подгруппа $U$ регулярна на множестве $\Omega \backslash\{\alpha\}$; в частности, $G=B\langle v\rangle B=$ $B\langle v\rangle U$, дде $v \in G \backslash B$

4) зруппа $G$ есть $Z$-группа на $\Omega$.

ДокАЗАТЕЛЬСтво. 1) По лемме 2.2 подгруппа $B$ сильно вложена в $G$, и по лемме 1.1 для любой инволюции $j \in G \backslash B$ подгруппа $T=B \cap B^{j}$ не содержит инволюций и транзитивна на множестве всех инволюций групшы $U$. Учитывая лемму 2.2 и определение групшы Фробениуса [12], заключаем, что $T^{u}=H$ для подходящего $u \in U$ и $j^{u}$ инвертирует $H$. Ввиду утверждения 6) леммы 2.2 все инволюции, инвертирующие $H$, содержатся в $H v=v^{H}$. Следовательно, $j \in v^{B}$ и утверждение доказано.

2) Очевидно, что $B=G_{\alpha}$. Ввиду утверждения 1) $B$ транзитивна на $\Omega \backslash\{\alpha\}$ и, следовательно, $G$ - 2-транзитивна на $\Omega$. Из лемм $1.1,1.2,2.2$ вытекает, что для любого $g \in G \backslash B$ пересечение $B \cap B^{g}$ совпадает с подходящим неинвариантным множителем $\Phi$ робениуса $H^{u}$ группы $B$, здесь $u \in U$. Это означает, что $H=G_{\alpha \beta}$ - стабилизатор двух точек. Утверждение доказано.

3) Так как $G_{\alpha}=U \lambda G_{\alpha \beta}$ и $B$ транзитивна на $\Omega \backslash\{\alpha\}$, то $U$ регулярна на $\Omega \backslash\{\alpha\}$. Отсюда также следует равенство $G=B\langle v\rangle B=B\langle v\rangle U$ для любого $v \in G \backslash B$. Утверждение доказано.

4) Пусть $\gamma \in \Omega \backslash\{\alpha, \beta\}$ и $u \in U$ элемент такой, что $\beta^{u}=\gamma$. Поскольку $H$ - неинвариантньй множитель групшы Фробениуса $B$, а $u$ - неединичный элемент из ее ядра $U$, то $H \cap H^{u}=1$. Следовательно, $G_{\alpha \beta \gamma}=1$ как подгруппа пересечения $H \cap H^{u}$. Ввиду 2 -транзитивности $G$ заключаем, что ее стабилизатор любых трех точек из $\Omega$ тривиален, т.е. $G-Z$-группа [7]. Лемма доказана.

Лемма 2.4. Пусть $B$ - группа Фробениуса с неинвариантным множителем $H$ и ядром $U$ и $H=B \cap B^{v}$, где $v-$ инволюиия из $G \backslash B$. Тогда

1) любой әлемент $g \in G \backslash B$ имеет единственное представление в виде $g=u_{1} x v u_{2}$, где $u_{1}, u_{2} \in U, x \in H$

2) элемент $j \in G \backslash B$ есть инволюиия тогда и только тогда, когда $j=u^{-1} \times$ $h^{-1} v h u=u^{-1} h^{-2} v u$, дде $u \in U, h \in H-$ однозначно определенные для $j$ әлементы;

3) если $i, j$ - различные инволючии из $G u|i j| \neq 2$, то әлемент $i j$ сопряжен $c$ әлементом $k v$, где $k$ - инволюиия из $U$;

4) если $k \in U, v \in G \backslash B-$ инволючии, то $A=C_{G}(k v)$ - сильно изолированная периодическая абелева подгруппа, инвертируемая инволючиями $k, v$, $N_{G}(A)=A \lambda T$ - әруппа Фробениуса с неинвариантным множителем $T=$ $U \cap N_{G}(A)$ и $A \cap A^{g}=1$ для любого әлемента $g \in G \backslash N_{G}(A)$.

ДокАЗАтЕльство. 1) Существование такого разложения для $g$ следует из равенства $G=B\langle v\rangle U$ (лемма 2.3). Пусть $g=u_{1} x v u_{2}=u_{3} y v u_{4}$, где $u_{1}, u_{2}, u_{3}, u_{4} \in U, x, y \in H$. Тогда $1=u_{1} x v u_{2} u_{4}^{-1} v y^{-1} u_{3}^{-1}, x^{-1} u_{1}^{-1} u_{3} y=v u_{2} u_{4}^{-1} v \in B \cap U^{v}$. Следовательно, 
$u_{2}=u_{4}$ и $u_{1} x=u_{3} y$. Так как $H \cap U=1$, то $x=y$ и $u_{1}=u_{3}$. Это означает, что разложение $g=u_{1} x v u_{2}$ единственно. Утверждение доказано.

2) По лемме 2.3 множество всех инволюций из $G \backslash B$ совпадает с множеством $v^{B}$ и второе утверждение леммы вытекает из первого.

3) Ввиду леммы 2.2 можно считать, что $i \in B$, и утверждение 1) леммы 2.3 завершает доказательство.

4) Из лемм 1.1, 1.2, 2.2 легко следует, что $A$ не содержит инволюций. Далее, $k, v \in$ $N_{G}(A), C_{A}(k)=1$ и по леммам $2.2,1.1 k$ - конечная инволюция. Следовательно, для любого элемента $a \in A^{\#}$ порядок элемента $k k^{a}$ конечен и нечетен, $a \in\left\langle k k^{a}\right\rangle$ и $a^{i}=a^{-1}$. Это означает, что $A$ - периодическая абелева группа, инвертируемая инволюциями $k$ и $v$. Понятно, что $A$ сильно изолирована в $G$ и $k^{A}=A k$-множество всех инволюций из $N_{G}(A)=L$. Отсюда вьводим, что $L=A \lambda C_{L}(k)$ - группа Фробениуса, поскольку $C_{L}(k)=L \cap U$. Ввиду сильной изолированности в $G$ подгруппа $A$ пересекается со своими сопряженными подгрупшами тривиально. Лемма доказана.

Лемма 2.5. Пусть $B$ - группа Фробениуса с неинвариантным мнохителем $H$ и ядром $U$ и $H=B \cap B^{v}$, где $v$ - инволюиия из $G \backslash B$. Тогда в группе $G$ выполняется структурное тождество Судзуки

$$
v t v=u^{-1} v u, \quad t, u \in U^{\#}, \quad t^{2}=1, \quad t \in\langle u\rangle, \quad|t v|=2 n+1<\infty .
$$

При этом для фиксированных $U, H, v$ существует единственная пара әлементов $t, u$ с таким свойством.

ДокАЗАТЕЛЬСТво. Пусть $i \in U$ - произвольная инволюция. По лемме 2.4 viv $=$ $y^{-1} x v x^{-1} y$, где $y \in U, x \in H$. Если $x=1$, полагаем $i=t, u=y$. Пусть $x \neq 1$. Тогда ввиду леммы 2.2 и выписанного вьше соотношения имеем

$$
v t v=v x i x^{-1} v=x^{-1}(v i v) x=x^{-1}\left(y^{-1} x v x^{-1} y\right) x=y^{-x} v y^{x}=u^{-1} v u .
$$

По лемме 1.3 выполняется равенство $(v t)^{u}=(v t)^{2}$, порядок элемента $v t$ конечен и нечетен, $u \in U$ и $t \in\langle u\rangle$.

Покажем, что пара $t, u$ единственна. Действительно, если $z$ - произвольная, отличная от $t$ инволюция из $B$, то $z=h t h^{-1}$ для некоторого неединичного элемента $h$ из $H=B \cap B^{v}$, инвертируемого инволюцией $v$ (лемма 1.2). Отсюда

$$
v z v=v h t h^{-1} v=h^{-1}(v t v) h=h^{-1}\left(u^{-1} v u\right) h=u^{-h} h^{-1} v h u^{h},
$$

причем такое представление для элемента $v z v$ единственно (лемма 2.4). В силу леммы $2.2 h u^{h} \notin U$ и, следовательно, существует ровно одна пара элементов $t \in Z(U)$, $u \in U$, для которых вьполняется соотношение $v t v=u^{-1} v u$. Лемма доказана.

3. Доказательство теоремы 1. На протяжении данного пункта группа $G$ удовлетворяет условиям теоремы $1, U$ - ее сильно изолированная 2 -подгруппа ранга не меньшего 2 и $B=N_{G}(U)$. Легко убедиться, что для группы $G$ и ее подгрупп $U$ и $B$ справедливы леммы 1.1-1.3, 2.2-2.5. 
ЛЕмма 3.1. В әруппе $G$ для некоторых инволюиий $v \in G \backslash B u t \in U$ выполняется структурное тохдество Судзуки

$$
v t v=t v t
$$

При этом для инволючии $v$ существует в точности одна такая инволющия $t \in U$.

ДокАЗАТЕЛЬСтво. По лемме 2.2 все инволюции группы $G$ сопряжены и, следовательно, $C_{G}(t)$ есть 2 -группа для каждой инволюции $t$ из $G$. Отсюда выводим, что подгруппа порядка 6 из условий теоремы есть группа диэдра, и теперь утверждение вытекает из лемм 1.3, 2.5. Лемма доказана.

Пусть $v$ - инволюция из леммы 3.1. Обозначим $H=B \cap B^{v}, Z=\Omega_{1}(Z(U))$ и $B_{0}=Z \lambda H$. По лемме $2.2 B_{0}$ изоморфна подгрупше Бореля группы $L_{2}(Q)$ над локально конечным полем $Q$ характеристики 2 .

ЛЕмма 3.2. Группа $G_{0}=B_{0}\langle v\rangle B_{0}$ есть подгруппа в $G$, изоморфная $L_{2}(Q)$.

ДокАЗАтЕльство. Покажем вначале, что $G_{0}$ - подгруппа в $G$. Понятно, что $G_{0}=G_{0}^{-1}$, и нужно доказать, что $G_{0} \cdot G_{0} \subseteq G_{0}$. Очевидно, что $G_{0}=B_{0} \cup B_{0} v Z$ и $G_{0} \cdot G_{0} \subseteq G_{0} \cup B_{0} v Z v Z$. Применим далее прием из [4].

По лемме 2.2 подгруппа $H$ регулярна на $Z^{\#}$, и если $z$ - произвольная инволюция из $Z$, то существует единственньй элемент $h \in H$ такой, что $z=t^{h}$. Поэтому

$$
v z v=v h^{-1} t h v=h v t v h^{-1}=h t v t h^{-1} \in G_{0}
$$

и ввиду произвольности элемента $z \in Z$ имеем $v Z v \subseteq G_{0}$. Следовательно, $G_{0} \cdot G_{0}=G_{0}-$ подгрупша группы $G$.

Далее, представим поле $Q$ в виде объединения $Q=\cup Q_{i}$ возрастающей цепочки конечных подполей

$$
Q_{1} \subseteq Q_{2} \subseteq Q_{3} \subseteq \cdots,
$$

а группы $H, B_{0}$ и $Z$ в виде объединениий $H=\cup H_{i}, B_{0}=\cup B_{i}, Z=\cup Z_{i}$ соответствующих цепочек конечных подгрупп:

$$
H_{1}<H_{2}<H_{3}<\cdots, \quad B_{1}<B_{2}<B_{3}<\cdots, \quad Z_{1}<Z_{2}<Z_{3}<\cdots,
$$

где $Z_{i}=Z \cap B_{i}$ и $B_{i}$ изоморфна подгрупе Бореля группы $L_{2}\left(Q_{i}\right)$. При этом, очевидно, можно считать, что $t \in Z_{1}$. Этим цепочкам соответствует цепь конечных подмножеств

$$
G_{1} \subseteq G_{2} \subseteq G_{3} \subseteq \cdots
$$

где $G_{i}=B_{i}\langle v\rangle B_{i}$. Теперь, используя те же рассуждения, что и вьше, и инвертируемость подгрупп $H_{n}$ инволюцией $v$ (лемма 2.2 ), докажем, что $G_{n}$ - конечные подгрупш, изоморфные по теореме Цассенхауза [16] (см. также [7, теорема 3.18]) $L_{2}\left(Q_{n}\right)$. Это означает, что $G_{0}=\cup G_{n}$ - локально конечная подгруппа, причем ввиду леммы 2.2 подгруппа $B_{0}=G_{0} \cap B$ сильно вложена в $G_{0}$. По теореме 3 из [11] $G_{0} \simeq L_{2}(Q)$. Заметим также, что в этом изоморфизме можно убедиться из других соображений, поскольку ввиду лемм $2.2,3.1$ умножение в $G$ однозначно определяется строением подгруппы $B_{0}$, действием $v$ на $H$ и структурньм тождеством Судзуки (2). Лемма доказана. 
Лемма 3.3. Пусть $у \in U u y^{2}=t$, где $t$ - инволюиия из структурного тождества Судзуки (2). Тогда имеет место соотношение

$$
v y v=y_{1} k v y_{1}^{-1} y^{-1}
$$

для некоторых әлементов $y_{1} \in U u k \in H$.

ДокАЗАТЕЛЬСтво. По лемме 2.4 элемент vyv имеет единственное разложение вида

$$
v y v=y_{1} k v y_{2}
$$

где $y_{1}, y_{2} \in U, k \in H$. Нам нужно доказать, что $y_{2}=y_{1}^{-1} y^{-1}$. Из равенств (3) и $k^{v}=k^{-1}$ (лемма 2.2) вытекают соотношения

$$
v y_{1} v=y v y_{2}^{-1} k, \quad v y_{2} v=k^{-1} y_{1}^{-1} v y
$$

Перемножая эти равенства, получаем

$$
v y_{1} y_{2} v=y v y_{2}^{-1} y_{1}^{-1} v y
$$

По условию леммы $|y|=4$, ввиду утверждения 2) леммы $2.4 y_{1} y_{2} \neq 1$ и по лемме 2.2 $v y_{1} y_{2} v \notin B$. По утверждению 1) леммы $2.4 v y_{1} y_{2} v=w h v z$, где $w, z \in U, h \in H$, и в силу соотношения (4) выполняется $w h v z=y z^{-1} v h^{-1} w^{-1} y$. По утверждению 1) леммы $2.4 z=w^{-1} y$ и $y=w z$. Далее, ввиду (3) имеем

$$
t v t=v t v=v y^{2} v=y_{1} k v y_{2} \cdot y_{1} k v y_{2}=y_{1} v\left(y_{2} y_{1}\right)^{k} v y_{2}, \quad v\left(y_{2} y_{1}\right)^{k} v=y_{1}^{-1} t v t y_{2}^{-1}
$$

Заметим, что $\left|y_{2} y_{1}\right|=\left|y_{1} y_{2}\right|=4$, так как если $y_{1} y_{2}$ - инволюция, то $w z=1$ (утверждение 2) леммы 2.4), что противоречит полученному вьше равенству $w z=y$. Поэтому $\left(y_{2} y_{1}\right)^{2}$ - инволюция и, учитывая, что $t \in Z=Z(U)$, из равенств (5) и $v y_{1} y_{2} v=w h v z$ мы выводим

$$
v\left(y_{2} y_{1}\right)^{2 k} v=y_{1}^{-1} t v t y_{2}^{-1} \cdot y_{1}^{-1} t v t y_{2}^{-1}=y_{1}^{-1} t v\left(y_{1} y_{2}\right)^{-1} v t y_{2}^{-1}=y_{1}^{-1} t z^{-1} h v w^{-1} t y_{2}^{-1}
$$

Согласно утверждению 2) леммы $2.4 w^{-1} t y_{2}^{-1} \cdot y_{1}^{-1} t z^{-1}=w^{-1} y_{2}^{-1} y_{1}^{-1} z^{-1}=1$, откуда $y_{2}^{-1} y_{1}^{-1}=w z=y$ и $y_{2}=y_{1}^{-1} y^{-1}$. Как отмечалось вьше, это доказьвает лемму.

Пусть $i$ - инволюция из $Z, v$ - инволюция из леммы $2.2, x=i v$ и $A=C_{G}(x)$.

Лемма 3.4. Подгруппа $A$ - периодическая локально ииклическая группа. 
ДокАЗАТЕЛЬСТво. По лемме $2.4 A$ - сильно изолированная периодическая абелева подгрупша и $N_{G}(A)=A \lambda T$ - группа Фробениуса, причем $T=N_{G}(A) \cap U$. Если $A \cap H^{g} \neq 1$ для некоторого $g \in G$, то из леммы 2.2 вытекает равенство $A=H^{g}$ и локальная цикличность группы $A$.

Пусть подгруппа $A$ взаимно проста со всеми подгруппами $H^{g}, p \in \pi(A)$ и $P=$ $\Omega_{1}\left(O_{p}(A)\right)$ - нециклическая подгруппа. Ввиду леммы 2.4 можно считать, что $a=i v \in P$. Пусть $b \in P^{\#}-$ произвольный элемент. Так как $b=j v$, где $j-$ инволюция из $v^{A}$, то по лемме 2.4 для некоторого элемента $g \in G$ имеет место равенство $j^{g} v^{g}=k v$, где $k \in Z$. Поскольку $a, k v \in G_{0} \simeq L_{2}(Q)$ (лемма 3.2), то подгрупшы $\langle a\rangle,\langle k v\rangle$ сопряжены в $G_{0}$. Учитывая лемму 2.4 , приходим к выводу, что подгруппы $\langle a\rangle$ и $\langle b\rangle$ сопряжены в подгруппе $N_{G}(A)$.

Таким образом, $T$ действует транзитивно на множестве всех циклических подгрупा из $P$. Как локально конечная 2-групша периода 4 с единственной подгруппой порядка 2 , $T$ - либо циклическая группа порядка 4, либо группа кватернионов порядка 8 [17, теорема 12.5.2]. Поскольку число различных циклических подгрупп из $P$, равное $(|P|-1) /(p-1)$, не меньше, чем $p+1 \geqslant 4$, и инволюция из $T$ оставляет каждую циклическую подгруппу из $P$ на месте, то $T$ - группа кватернионов порядка 8 , а $P$ группа порядка 9. Так как группа автоморфизмов групшы простого порядка абелева, то $\pi(A)=3, A_{0}=A \cap G_{0}-$ квазищиклическая 3 -групша и $A$ - прямое произведение двух квазициклических 3-подгрупп. Покажем, что данный случай невозможен.

Рассмотрим в поле $Q$ два конечных подполя $R \subseteq F$ порядков $2^{n}>4$ и $2^{n p}, n>1$, $p$ - простое, и подгрупшы $L_{2}(R), L_{2}(F)$ из $G_{0}$. Ввиду доказанного выше $3 \notin \pi(H)$ и циклические подгруппы порядков $2^{n}+1,2^{n p}+1$ из $L_{2}(R)$ и $L_{2}(F)$ содержат 3 -элементы, сопряженные с элементами из $A$. Поэтому $2^{n}+1=3^{k}, 2^{n p}+1=3^{m}$, и поскольку $\left(3^{k}-1\right)^{2}=3^{2 k}-2 \cdot 3^{k}+1 \neq 3^{m}-1$, то $p>2$. Но тогда

$$
3^{m}-1=\left(3^{k}-1\right)^{p}=3^{k p}-p 3^{k(p-1)}+\cdots+p 3^{k}-1
$$

и $3^{m}=3^{k}(3 s+p)$. Из последнего равенства получаем, что $p=3$,

$$
3^{m}=\left(3^{k}-1\right)^{3}+1=3^{3 k}-3^{2 k+1}+3^{k+1}
$$

и $3 k=2 k+1$. Отсюда $k=1$ и $2^{n}=2<4$. Противоречие, и лемма доказана.

Лемма 3.5. Имеет место равенство $G=G_{0} \simeq L_{2}(Q)$.

ДоКАЗАТЕЛЬСТво. Очевидно достаточно доказать, что $U=Z$. Предположим противное, и пусть $y$ - элемент порядка 4 из $U$ такой, что $y^{2}=t$, где $t$ - инволюция из леммы 3.1. Тогда по лемме $3.3 v y v=y_{1} k v y_{1}^{-1} y^{-1}$ и $v y v y^{-1}=z t=a$, где $z=y_{1} k v y_{1}^{-1}$ инволюция. Инволюции $v, t, z$ и $y v y^{-1}$ инвертируют $a, x=t v$ централизует $a$. Следовательно, $a \in A=C_{G}(x)$.

Покажем, что $A \leqslant G_{0}$. Если $A \neq A_{0}=A \cap G_{0}$, то в разности $A \backslash A_{0}$, очевидно, найдется элемент $b$ такой, что пересечение $\pi(\langle b\rangle) \cap \pi\left(A_{0}\right)$ непусто. По лемме 2.4 инволюция $v$ инвертирует $A$, следовательно, $b$ есть произведение двух инволюций. По лемме $2.4 b$ сопряжен с некоторым элементом $v i \in G_{0}$, где $i \in Z$, и ввиду изоморфизма $G_{0} \simeq L_{2}(Q)$ (лемма 3.2), свойств групा $L_{2}(Q)$ (см., например, [9]) и выбора элемента $b$ элемент $v i$ сопряжен в $G_{0}$ с некоторьп элементом из $A_{0}$. Но тогда $A_{0}$ содержит 
циклическую подгрупшу порядка $|b|$ и из локальной цикличности $A$ (лемма 3.4 ) следует $b \in A_{0}$. Полученное противоречие означает, что $A \leqslant G_{0}$.

Итак, $A<G_{0}$ и, значит, $y v y^{-1}=v a \in G_{0}$. По лемме $2.4 y v y^{-1}=(h v)^{w}$ для некоторых $h \in H, w \in Z$, причем понятно, что $h \neq 1$, так как в противном случае $1 \neq w y \in C_{U}(v)$, что противоречит сильной изолированности $U$ в $G$. Представим элемент $h$ нечетного порядка в виде $h=h_{1}^{2}, h_{1} \in\langle h\rangle$. Тогда из равенства $y v y^{-1}=(h v)^{w}$ выводим $y v y^{-1}=(h v)^{w}=\left(h_{1}^{2} v\right)^{w}=w h_{1} v h_{1}^{-1} w$, и элемент $v$ перестановочен с неединичньм элементом $y^{-1} w h_{1}$ из $B$. Полученное противоречие означает, что элемента $y \in U$ со свойством $y^{2}=t$ не существует, $U=Z$ и ввиду леммы $2.3 G=G_{0}$. Лемма доказана.

Теперь теорема 1 следует из лемм 2.1 и 3.5 .

4. 2-группы Судзуки $S(P, x)$. Нам понадобятся некоторые свойства группы $U=$ $S(P, x)$, доказательства которых, приведенные в [5], справедливы для произвольного локально конечного поля $P$ характеристики 2 .

ЛЕмма 4.1. Справедливы следующие утверэдения:

1) $U-$ zрупnа периода 4 ;

2) $Z=\{(0, \alpha) \mid \alpha \in P\}-$ иентр группы $U$;

3) $Z$ - элементарная абелева группа и все инволюиии из $U$ содержатся в $Z$;

4) если $t_{0}=(1,0)$, то $C_{U}\left(t_{0}\right)=\left\{(\alpha, \beta) \mid \alpha, \beta \in P, \alpha^{x}=\alpha\right\}$;

5) для любого әлемента $t \in U \backslash Z$ смежний класс $t Z$ состоит в точности из всех решений уравнения $y^{2}=t^{2}$ группь $U$;

6) $U$ не содержит группы кватернионов;

7) для кахдого $0 \neq \lambda \in P$ отобрахсение $\varphi(\lambda):(\alpha, \beta) \Rightarrow\left(\lambda \alpha, \lambda^{1+x} \beta\right)$ является автоморфизмом группь $U$;

8) если $\Phi=\{\varphi(\lambda) \mid 0 \neq \lambda \in P\}$, то $\Phi$ изоморфна мультипликативной әруппе поля $P$.

Обозначим через $M(P, x)$ - расширение групшы $U=S(P, x)$ с помощью группы $\Phi$. Для доказательства теоремы нам понадобятся свойства расширений более общего вида. Предположим, что $U$ допускает периодическую нечетную локально циклическую группу автоморфизмов $H$, действующую регулярно на множестве $Z^{\#}$ всех инволюций из $U$, и пусть $B=U \lambda H-$ расширение $U$ с помощью $H$. Обозначим также $z_{0}=t_{0}^{2}=$ $(1,0)^{2}=(0,1)$.

ЛЕмма 4.2. Справедливы следующие утверәсдения:

1) $B$ - группа Фробениуса;

2) зруппа $H$ действует регулярно на множестве неединичных әлементов факторгруппь $U / Z$;

3) группа $Z \lambda H$ и факторгруппа $B / Z$ изоморфны подгруппе Бореля группы $L_{2}(Q)$ над подходящим локально конечным полем $Q$ характеристики 2.

ДокАЗАтЕльство. 1) Утверждение очевидно (см., например, [14]).

2) Пусть $b \in U$ и $|b|=4$. Ввиду условий леммы для некоторого $h \in H$ выполняется равенство $z_{0}^{h}=b^{2}$. По лемме $4.1 t_{0}^{h} Z=b Z$, что доказывает утверждение.

3) Утверждение вытекает из утверждения 2) и леммы 4.4 из [4]. Лемма доказана. 
Пусть $Q$ - поле из утверждения 3 ) леммы 4.2. Представим его в виде объединения $Q=\cup Q_{i}$ возрастающей цепочки конечных подполей

$$
Q_{1} \subseteq Q_{2} \subseteq Q_{3} \subseteq \cdots
$$

а подгрупшы $H$ и $Z$-в виде объединениий $H=\cup H_{i}, Z=\cup Z_{i}$ соответствующих цепочек конечных подгрупп

$$
\begin{gathered}
H_{1}<H_{2}<H_{3}<\cdots, \\
Z_{1}<Z_{2}<Z_{3}<\cdots,
\end{gathered}
$$

где $Z_{k} \lambda H_{k}$ изоморфна подгруппе Бореля группы $L_{2}\left(Q_{k}\right)$ (возможность этих представлений вытекает непосредственно из утверждения 3) леммы 4.2).

ЛЕмма 4.3. Группа $B$ представима в виде обгединения $B=\cup B_{k}$ возрастающей иепочки $B_{1}<B_{2}<B_{3}<\cdots$ конечных подгрупп $B_{k}$, изоморфных $M\left(Q_{k}, x_{k}\right)$, где $Q_{k}$ - конечные поля чепочки (6).

ДокАЗАТЕЛЬСтво. Очевидно можно считать, что $z_{0} \in Z_{1}$. Так как $H$ действует регулярно на множествах неединичных элементов из $Z$ и $U / Z$, а группа $U$ неабелева (леммы 4.1, 4.2), то, начиная с некоторого номера $m$, подгрупшы $U_{k}=\left\langle t_{0}^{H_{k}}\right\rangle$ неабелевы. Без ограничения общности считаем, что уже $U_{1}=\left\langle t_{0}^{H_{1}}\right\rangle$ - неабелева подгрупа. Обозначим $\left|Q_{k}\right|=q_{k}$. Тогда $\left|Z_{k}\right|=q_{k},\left|H_{k}\right|=q_{k}-1$ и ввиду изоморфности факторгрупшы $B / Z$ подгруппе Бореля из $L_{2}(Q)$ (лемма 4.2 ) факторгруппа $U_{k} Z / Z$ имеет порядок $q_{k}$.

По лемме 4.1 в подгрупше $U$ любой смежньй класс $u Z$, отличньй от $Z$, состоит в точности из всех решений уравнения $x^{2}=u^{2}$. Поэтому в факторгрупе $\bar{U}=U / Z_{k}$ справедливо равенство $\left|\Omega_{1}(\bar{U}): \bar{Z}\right|=q_{k}$. Из обобщенной теоремы Машке [18] следует $\Omega_{1}(\bar{U})=\bar{Z} \times \bar{U}_{k}$. Таким образом, подгрупша $U_{k}$ неабелева, допустима относительно $H_{k}$, имеет порядок $q_{k}^{2}$ и $H_{k}$ действует регулярно на множестве ее инволюций. Как вытекает из теоремы Хигмана [6] (см. также [5, с. 115]), $U_{k}$ изоморфна некоторой 2-группе Судзуки $S\left(Q_{k}, x_{k}\right)$, где $x_{k}$ - подходящий автоморфизм поля $Q_{k}$. По лемме 6 из [5] $B_{k}=U_{k} \lambda H_{k}$ изоморфна группе $M\left(Q_{k}, x_{k}\right)$.

Ввиду определения подгрупп $U_{k}$ и $B_{k}$ имеем $U_{k}<U_{k+1}$ и $B_{k}<B_{k+1}$. Таким образом, цепочке (6) сответствуют цепочки подгрупп

$$
\begin{aligned}
& U_{1}<U_{2}<U_{3}<\cdots, \\
& B_{1}<B_{2}<B_{3}<\cdots,
\end{aligned}
$$

при этом $B_{k} \simeq M\left(Q_{k}, x_{k}\right)$. Обьединение $L$ цепочки (9) содержит подгрупшы $H, Z$ и нетривиально пересекается с множеством $U \backslash Z$. Учитьвая действие подгрупы $H$ на $U / Z$ (лемма 4.2), заключаем, что $L=B$. Лемма доказана.

5. Доказательство теоремы 2 и следствия 1. Итак, пусть группа $G$ удовлетворяет условиям теоремы 2 и предположим, что $B=N_{G}(U) \neq G$. Поскольку $r(U)>1$, то в рассматриваемой ситуации верны леммы 2.2-2.5, 4.1-4.3. Воспользуемся стандартными, ранее введенными обозначениями. 
Лемма 5.1. В группе $G$ выполняется структурное тождество Судзуки

$$
v t v=u^{-1} v u, \quad t, u \in U^{\#}, \quad t^{2}=1, \quad t \in\langle u\rangle, \quad|t v|=2 n+1<\infty .
$$

При этом для фиксированных $U, H, v$ существует единственная пара әлементов $t$, и с таким свойством.

ДокАЗАТЕЛЬСТво. По лемме 2.5 для инволюции $v$ существует такая пара элементов $t, u \in U$. Учитьвая теорему 1 , заключаем, что $t \neq u$ и $|u|=4$. Лемма доказана.

Ввиду леммы 4.3 без ограничения общности можно считать, что элементы $t, u$ из структурного тождества Судзуки (10) содержатся во всех подгрупшах $U_{n}$ цепочки (8). Пусть $H_{n}, U_{n}, B_{n}=U_{n} \lambda H_{n}$ - подгруппы цепочек (7)-(9).

Лемма 5.2. Каждый әлемент из $U_{n}^{\#}$ сопряжен әлементом из $H_{n}$ точно $к$ одному из әлементов $u^{2}, u, u^{-1}, \pi(k)=u k^{-1} u^{-1} k\left(1 \neq k \in H_{n}\right)$, әде $u$ - әлемент порядка 4 из структурного тождества Судзуки (10).

ДокАЗАТЕЛЬство. Лемма доказана в [5, предложение 20].

По лемме 2.4 если $1 \neq x \in U$, то

$$
v x v=g(x) h(x) v f(x),
$$

где $g(x), f(x)$ - функции, определенные на $U^{\#}$ со значениями в $U, h(x)$ - функция, отображающая $U^{\#}$ в $H$ (см. [5, с. 109]).

ЛЕмма 5.3. Пусть $\pi(k)=u k^{-1} u^{-1} k$, əде $1 \neq k \in H_{n}$. Тогдa

$$
f(\pi(k))=r u r^{-1} k u^{-1} k^{-1}, \quad g(\pi(k))=u k^{-2} r u^{-1} r^{-1} k^{2}, \quad h(\pi(k))=k^{-2} r^{2},
$$

әде $r$ - әлемент из $H_{n}$ такой, что $t^{r}=\left[k, t^{k}\right]$.

ДокАЗАТЕЛЬСТво. Воспользуемся вычислениями предложения 20 из [5]. Учитывая структурное тождество (10) и соотношение $k^{v}=k^{-1}$ (лемма 2.2), получаем $v u k^{-1} u^{-1} k v=v u v v k^{-1} v v u^{-1} v v k v=u v t k t v u^{-1} k^{-1}=u k^{-2} v k^{-1}\left(k^{-1} t k t\right) k v k u^{-1} k^{-1}$.

Очевидно, что элемент $k^{-1} t k t \in Z\left(U_{n}\right)=Z_{n}$, и, так как $k \neq 1$, то существует единственный элемент $r=r(k) \in H_{n}$ такой, что $k^{-1}\left(k^{-1} t k t\right) k=r^{-1} t r$. Таким образом,

$$
v(\pi(k)) v=v u k^{-1} u^{-1} k v=u k^{-2} r v t v r^{-1} k u^{-1} k^{-1}=u k^{-2} r u^{-1} v u r^{-1} k u^{-1} k^{-1} .
$$

По определению функций $f, g$ и $h$ и лемме 2.4 мы имеем

$$
f(\pi(k))=r u r^{-1} k u^{-1} k^{-1}, \quad g(\pi(k))=u k^{-2} r u^{-1} r^{-1} k^{2}, \quad h(\pi(k))=k^{-2} r^{2} .
$$

Лемма доказана.

Пусть $B_{n}$ - член цепочки (9). 
Лемма 5.4. Группа $G_{n}=B_{n}\langle v\rangle B_{n}-$ конечная подгруппа группь $G$.

ДокАЗАТЕЛЬСТво. Очевидно, что $G_{n}=G_{n}^{-1}$, и остается установить замкнутость умножения в $G_{n}$. Так как $G_{n} \cdot G_{n}=B_{n} v U_{n} v U_{n}$, то ввиду равенства (11) достаточно показать, что $g(x), f(x), h(x) \in B_{n}$ при любом $1 \neq x \in U_{n}$. Докажем это.

Если $x$ - один из элементов $t, u, u^{-1}$, то доказываемое включение следует непосредственно из структурного тождества (10) и определения функций $g, f, h$. Действительно, из $v t v=u^{-1} v u$ вытекают равенства $v u v=u v t, v u^{-1} v=t v u^{-1}$ и элементы

$$
f(u)=t, \quad g(u)=u, \quad h(u)=1, \quad f\left(u^{-1}\right)=u^{-1}, \quad g\left(u^{-1}\right)=t, \quad h\left(u^{-1}\right)=1
$$

принадлежат $B_{n}$ при любом $n$.

Далее, из леммы 5.3 следует, что $f(\pi(k)), g(\pi(k)), h(\pi(k)) \in B_{n}$ для любого элемента $k \in H_{n}^{\#}$. Следовательно, если $x=\pi(k)$ при некотором $k \in H_{n}^{\#}$, то $g(x), f(x), h(x) \in B_{n}$.

Заметим, что для любых элементов $y \in U_{n}, d \in H_{n}$ справедливо равенство

$$
v y^{d} v=d v y v d^{-1}=d f(y) h(y) v g(y) d^{-1}=d f(y) d^{-1} \cdot h(y) d^{2} \cdot v \cdot d g(y) d^{-1} .
$$

Поэтому если $g(y), f(y), h(y) \in B_{n}$, то и $g\left(y^{d}\right), f\left(y^{d}\right), h\left(y^{d}\right) \in B_{n}$. По лемме 5.2 элемент $x \in U_{n}^{\#}$ сопряжен с помощью некоторого элемента из $H_{n}$ с одним из элементов $t, u, u^{-1}, \pi(k)$. Отсюда и ввиду вьшшедоказанного заключаем, что $g(x), f(x), h(x) \in B_{n}$ для любого элемента $x \in U_{n}$. Следовательно, $G_{n} \cdot G_{n}=G_{n}$ и лемма доказана.

ЛЕмма 5.5. Имеет место изоморфизм $G_{n} \simeq S z\left(Q_{n}\right)$ и $B_{n}-$ подгруппа Бореля в этой әpynne.

ДоказАТЕЛьСтво. Лемма доказана в [11].

ЛЕмма 5.6. Имеет место изоморфизм $G \simeq S z(Q)$, где $Q-$ поле из леммы 4.2 .

ДокАЗАТЕЛЬСТво. По леммам $5.4,5.5$ групша $G_{n}$ изоморфна конечной группе Судзуки $S z\left(Q_{n}\right)$. Значит, $q_{n}=2^{2 m_{n}+1}$ и поле $Q_{n}$ обладает единственным автоморфизмом $\theta_{n}$ со свойством $\theta_{n}^{2}=2$. Отсюда следует, что и поле $Q=\bigcup_{n} Q_{n}$ обладает автоморфизмом $\theta$ с этим свойством.

Далее воспользуемся доказательством аналогичной леммы из [11]. Как известно [5], [9], группа $S z(Q)$ порождается всевозможньми матрицами вида

$$
\begin{gathered}
(\alpha, \beta)=\left(\begin{array}{cccc}
1 & 0 & 0 & 0 \\
\alpha & 1 & 0 & 0 \\
\alpha^{1+\theta}+\beta & \alpha^{\theta} & 1 & 0 \\
\alpha^{2+\theta}+\alpha \beta+\beta^{\theta} & \beta & \alpha & 1
\end{array}\right), \\
h=\left(\begin{array}{cccc}
h^{1+\theta^{-1}} & 0 & 0 & 0 \\
0 & h^{\theta^{-1}} & 0 & 0 \\
0 & 0 & h^{-\theta^{-1}} & 0 \\
0 & 0 & 0 & h^{-1-\theta^{-1}}
\end{array}\right), \quad w=\left(\begin{array}{llll}
0 & 0 & 0 & 1 \\
0 & 0 & 1 & 0 \\
0 & 1 & 0 & 0 \\
1 & 0 & 0 & 0
\end{array}\right),
\end{gathered}
$$

где $\alpha, \beta, h \in P, h \neq 0$ (здесь мы отождествили подгрупу $H$ с мультипликативной группой поля $Q$ ) и $\theta$ - автоморфизм поля $Q$ с $\theta^{2}=2$. Цепочке конечных полей $Q_{n}(6)$ ввиду лемм $4.2,4.3,5.4,5.5$ соответствует цепочка конечных групш Судзуки

$$
G_{1}<G_{2}<G_{3}<\cdots, \quad G_{n} \simeq S z\left(Q_{n}\right),
$$


и, как следует из лемм $2.3,4.3$, объединение членов этой цепочки совпадает с группой $G$. В силу леммы 5.5 и теоремы 8 из [5] при любом натуральном $n$ существует изоморфизм $\psi_{n}$ подгрупшы $G_{n}=B_{n}\langle v\rangle B_{n}$ на подгруппу $S z\left(Q_{n}\right)$, при котором

$$
\psi_{n}(t)=(0,1), \quad \psi_{n}(u)=(1,0), \quad \psi_{n}(h)=h, \quad \psi_{n}(v)=w .
$$

Понятно, что изоморфизм $\psi_{n}$ однозначно восстанавливается по образам $t, u, H, v$. Поэтому ограничение $\psi_{n+1}$ на $G_{n}$ совпадает с $\psi_{n}$, и мы можем следующим образом корректно определить отображение $\psi$ групшы $G$ на групшу $S z(Q)$ : если $g \in G_{n}$, то $\psi(g)=\psi_{n}(g)$. Легко убедиться, что $\psi$ - изоморфизм $G$ на $S z(Q)$. Лемма и вместе с ней теорема 2 доказаны.

Заметим, наконец, что в периодической группе любая инволюция конечна, и поэтому следствие 1 вытекает из теоремы 4.1 [4] и теоремы 2 настоящей статьи.

\section{СПИСОК ЦИТИРОВАННОЙ ЛИТЕРАТУРЫ}

[1] Коуровская тетрадь. Нерешенные вопросы теории групп. 6-14 издания. Новосибирск, 1978-1999.

[2] Созутов А. И. О группах с сильно вложенной подгруппой // Международная конференция "Симметрия в естествознании". Тезисы докл. Красноярск, 1998. С. 121-123.

[3] Созутов А. И., Сучков Н. М. О некоторых бесконечных кратно транзитивных группах // Международный алгебраический семинар, посвященный 70-летию каффедры высшей алгебры МГУ (10-12 февраля 1999г.). Тезисы докл. М., 1999. С. 52.

[4] Созутов А. И. О некоторых бесконечных группах с сильно вложенной подгруппой // Алгебра и логика. (в печати).

[5] Suzuki M. On a class of doubly transitive groups // Ann. of Math. 1961. V. 75 . № 1. P. 105-145.

[6] Higman G. Suzuki 2-groups // Illinois J. Math. 1963. V. 7. P. 79-96.

[7] Горенстейн Д. Конечные простые группы. М.: Мир, 1985.

[8] Созутов А. И. О группах с классом фробениусово-абелевых элементов // Алгебра и логика. 1995. Т. 34. №5. С. 531-549.

[9] Бусаркин В. М., Горчаков Ю. М. Конечные расщепляемые группы. М.: Наука, 1968.

[10] Созутов А.И., Сучков Н. М. О некоторых дважды транзитивных группах. Препринт № 17. Красноярск: ИВМ СО РАН, 1998.

[11] Созутов А.И., Сучков Н. М. О бесконечных $Z$-группах с заданными стабилизаторами точек. Деп. ВИНИТИ 09.03.99 № 700-В99. Красноярск: Краснояр. гос. архит.-строит. акад., 1999.

[12] Созутов А. И., Шунков В. П. Об одном обобщении теоремы Фробениуса на бесконечные группы // Матем. сб. 1976. Т. 100. №4. С. 495-506.

[13] Беляев В.В. Группы с почти регулярной инволюцией // Алгебра и логика. 1987. Т. 26. № 5. C. 531-535.

[14] Старостин А. И. О группах Фробениуса // Укр. матем. ж. 1971. Т. 23. № 5. С. 629-639.

[15] Каргаполов М.И., Мерзляков Ю. И. Основы теории групп. М.: Наука, 1977.

[16] Zassenhaus H. Kennzeichnung endlicher linearen Gruppen als Permutationsgruppen // Abh. Math. Sem. Hamburg. 1936. V. 11. P. 17-40.

[17] Холл М. Теория групп. М.: ИЛ, 1962.

[18] Сучков Н. М. Автоморфно факторизуемые группы // Алгебра и логика. 1979. Т. 18. № 4. C. $481-487$.

(А. И. Созутов) Красноярская государственная 